\title{
December 2015 Critical Care Case of the Month
}

\author{
Samir Sultan, DO \\ Banner University Medical Center Phoenix \\ Phoenix, AZ
}

\section{History of Present IIIness}

The patient is a 32-year-old woman who presented with flank pain for 3 days to an outside hospital. She was diagnosed with pyelonephritis and begun on ceftriaxone. She was discharged against medical advice on cephalexin.

She returned to the same hospital 3 days later by ambulance with labored breathing and weakness and was emergently intubated. She was transferred for ventilator management and respiratory failure.

\section{Past Medical History}

She has a long history of poorly controlled diabetes mellitus.

\section{Physical Examination}

She is orally intubated and sedated.

Vitals: Temperature $-100.9^{\circ} \mathrm{F}$, Blood Pressure $-117 / 75 \mathrm{~mm} \mathrm{Hg}$, Heart Rate -148 beats per minute, Respiratory Rate - 31 breaths/min, SpO2 - $88 \%$ on assist control of 30, tidal volume of $350 \mathrm{~mL}$, PEEP 15, and an FiO2 100\%.

There is scatted rhonchi and rales but the remainder of the physical examination is unremarkable.

\section{Radiography}

Her admission portable chest X-ray is shown in Figure 1. 


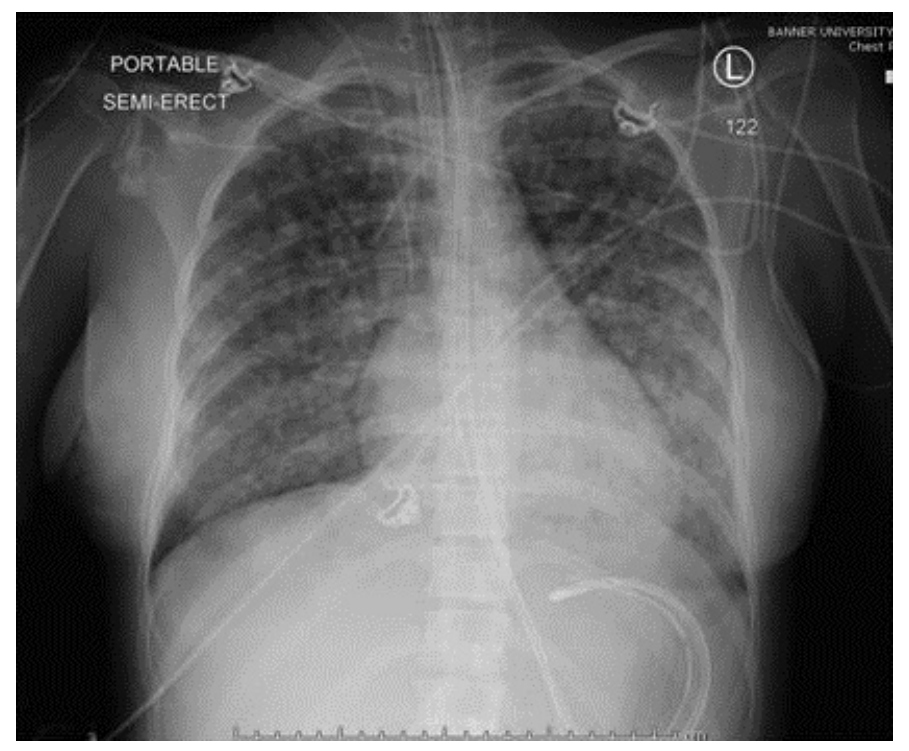

Figure 1. Admission portable AP of the chest.

Which of the following should be ordered as part of her initial work-up?

1. Administer broad spectrum antibiotics

2. Blood and urine cultures

3. Rapid influenza test

4. 1 and 3

5. All of the above 


\section{Correct! \\ 5. All of the above}

The patient's $\mathrm{SpO} 2$ is low on $100 \%$ oxygen and considerable positive end expiratory pressure (PEEP). Arterial blood gases are indicated to assess her oxygen status as we all as her acid-base status. Based on her history she likely has urosepsis and blood and urine cultures are indicated. As we enter influenza season it is important that influenza be considered on cases of diffuse pneumonia and respiratory failure.

The patient's arterial blood gases were pH 7.34, pO2 85, pCO2 35, HCO3- 18. Blood cultures showed no growth but a urine culture showed E. coli which was pan-sensitive to the test antibiotics. Rapid influenza test was positive for influenza B.

Which of the following are indicated next?

1. Begin oseltamivir (Tamiflu)

2. Biopsy of the lung with transbronchial biopsy or video-assisted thorascopic biopsy

3. Lower FiO2

4. 1 and 3

5. All of the above 


\section{Correct!}

\section{1 and 3}

Lung biopsy is not indicated at this time and has risk in this patient who is profoundly hypoxic on $100 \%$ FiO2. It is unclear whether she has the adult respiratory distress syndrome (ARDS) from sepsis or diffuse viral pneumonia from influenza. Oseltamivir is indicated but otherwise the therapy is supportive. Something does need to be done about her high FiO2. 100\% oxygen will eventually prove toxic to the lungs. The level that is safe is unclear but generally considered to be both concentration and timedependent. Most consider an $\mathrm{FiO} 2$ of $60 \%$ to be a reasonable goal.

At times the pO2 seems little affected by the $\mathrm{FiO} 2$, i.e., lowering the FiO2 results in a pO2 little different from the $\mathrm{pO} 2$ on an $\mathrm{FiO} 2$ of $100 \%$. The reason for this is that in ARDS there are two sets of alveoli, those that are ventilated and perfused and those that are not ventilated but perfused with the later causing the hypoxia. Lowering the FiO2 does not profoundly affect the alveoli that are both ventilated and perfused.

Unfortunately, lowering the $\mathrm{FiO} 2$ in this patient resulted in rapid desaturation.

Which of the following might be considered to lower of the FiO2?

1. Extracorporeal membrane oxygenation (ECMO)

2. Increase PEEP

3. Proning

4. 1 and 3

5. All of the above 


\section{Correct! \\ 5. All of the above}

All of these are reasonable to lower the FiO2 with proning and increasing PEEP being the simplest maneuvers. Increasing PEEP can result in barotrauma and usually levels above $15 \mathrm{~cm} \mathrm{H}_{2} \mathrm{O}$ result in no further $\mathrm{FiO} 2$ improvement. Proning may help if the lung is not evenly affected which can result in better ventilation-perfusion matching.

Unfortunately, both failed to increase the FiO2. There are a variety of other maneuvers such as increasing the expiratory time (which essentially results in increasing the PEEP) which was also unsuccessful.

Although initial trials were unimpressive, more recent trials suggest a possible mortality benefit with ECMO $(1,2)$. ECMO is done in specialized centers that have experience with this therapy. Given that this patient was young and functional a trial of ECMO seemed reasonable.

The patient was begun on venous to venous ECMO where venous blood is taken from a vein, oxygenated and returned to the venous system. This resulted in an improvement in the pO2 but also resulted in a depressed cardiac output and hypotension. The patient suffered a cardiac arrest but recovered spontaneous circulation. The ECMO was switched to venous-arterial (VA) ECMO where blood was removed from the femoral vein, oxygenated and returned to the arterial circulation through a catheter placed in the descending aorta through the femoral artery.

The patient developed an unusual appearance where the lower extremities were pink but the upper extremities and head were cyanotic.

What should be done next?

1. Administer esmolol

2. Consider moving the arterial catheter to another artery

3. Increase the flow rate from the ECMO

4. 1 and 3

5. All of the above 


\section{Correct! \\ 5. All of the above}

The patient has developed ECMO "harlequin" syndrome. This results from inadequate mixing of blood in the aorta where the relatively deoxygenated blood from the left heart is not adequately mixed with the oxygenated blood from ECMO (Figure 2).

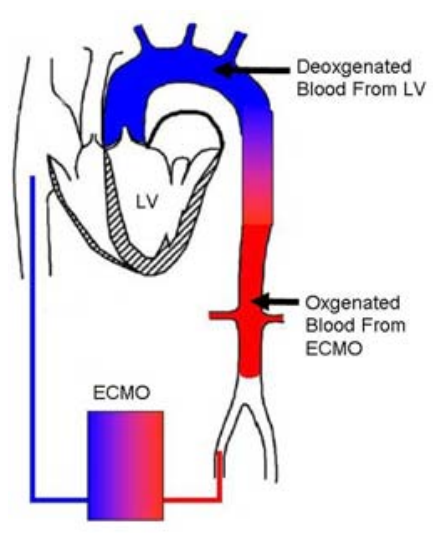

Figure 2. Schematic showing inadequate mixing of deoxygenated and oxygenated blood resulting in cyanosis in the upper extremities and head.

The deoxygenated blood is directed more towards the upper body while the oxygenated blood is directed more towards the lower extremities. Increasing the mixing or another mode of ECMO are strategies to deliver more oxygenated blood to the head and upper extremities. The flow rate of the ECMO was increased along with administration of esmolol to decrease the flow rate out of the left ventricle to allow more adequate mixing. If these had failed, moving the arterial line from the ECMO could be considered. Switching to another mode of ECMO such as venous-arterial-venous (VAV) where oxygenated blood is returned to both the arterial and venous circulation is another consideration.

The patient eventually improved after two days on ECMO and eventually was extubated and transferred to the floor.

\section{References}

1. Zapol WM, Snider MT, Hill JD, et al. Extracorporeal membrane oxygenation in severe acute respiratory failure. A randomized prospective study. JAMA. 1979;242(20):2193-6. [CrossRef] [PubMed]

2. Peek GJ, Mugford M, Tiruvoipati R, et al. Efficacy and economic assessment of conventional ventilatory support versus extracorporeal membrane oxygenation for severe adult respiratory failure (CESAR): a multicentre randomised controlled trial. Lancet. 2009;374(9698):1351-63. [CrossRef] [PubMed]

3. Moisan M, Lafargue M, Calderon J, Oses P, Ouattara A. Pulmonary alveolar proteinosis requiring "hybrid" extracorporeal life support, and complicated by acute necrotizing pneumonia. Ann Fr Anesth Reanim. 2013;32(4):e71-5. [CrossRef] [PubMed] 